\title{
Derivation Method for the Foundation Boundaries of Hydraulic Numerical Simulation Models Based on the Elastic Boussinesq Solution
}

\author{
Jintao Song, ${ }^{1,2,3}$ Chongshi Gu, ${ }^{1,2,3}$ Hao Gu, $^{1,2,3}$ and Shaowei Wang ${ }^{1,2,3}$ \\ ${ }^{1}$ State Key Laboratory of Hydrology-Water Resources and Hydraulic Engineering, Hohai University, Nanjing 210098, China \\ ${ }^{2}$ National Engineering Research Center of Water Resources Efficient Utilization and Engineering Safety, Hohai University, \\ Nanjing 210098, China \\ ${ }^{3}$ College of Water Conservancy and Hydropower, Hohai University, Nanjing 210098, China
}

Correspondence should be addressed to Chongshi Gu; zibet998@126.com

Received 25 September 2014; Revised 9 February 2015; Accepted 20 March 2015

Academic Editor: Rafael J. Villanueva

Copyright (C) 2015 Jintao Song et al. This is an open access article distributed under the Creative Commons Attribution License, which permits unrestricted use, distribution, and reproduction in any medium, provided the original work is properly cited.

\begin{abstract}
The foundation boundaries of numerical simulation models of hydraulic structures dominated by a vertical load are investigated. The method used is based on the stress formula for fundamental solutions to semi-infinite space body elastic mechanics under a vertical concentrated force. The limit method is introduced into the original formula, which is then partitioned and analyzed according to the direction of the depth extension of the foundation. The point load will be changed to a linear load with a length of $2 a$. Inverse proportion function assumptions are proposed at parameter $a$ and depth $l$ of the calculation points to solve the singularity questions of elastic stress in a semi-infinite space near the ground. Compared with the original formula, changing the point load to a linear load with a length of $2 a$ is more reasonable. Finally, the boundary depth criterion of a hydraulic numerical simulation model is derived and applied to determine the depth boundary formula for gravity dam numerical simulations.
\end{abstract}

\section{Introduction}

In hydraulic engineering, numerical simulation models are widely used in structural analysis [1], such as in infinite and discrete element methods. The finite element method (FEM) is the most widely used technique in hydraulic numerical simulation [2]. Selecting the foundation boundaries affects the number of finite element mesh size, calculation accuracy, and computation time [3]. Many scholars have proposed selection methods for foundation boundaries based on the finite element simulation model. Foundation boundaries are generally selected based on engineering experience [4] or by comparing the accuracy of stress results using FEM for different boundary conditions [5]. However, these selection methods are still not applied in engineering design because of different issues in analyzed objects and calculation accuracy [6]. At present, comprehensive qualitative studies remain inadequate in both China and abroad; for example, selecting calculation boundaries has not been studied based on elasticity theory [7]. According to Saint-Venant's principle [8], hydraulic structure load has more influence on the stress of a point near the load and minimal influence on the stress of a point far from the load; hence, conducting a partition discussion is necessary. Research on foundation boundaries under dam structure load can analyze foundation stress under loads and search for the minimum impact areas to function as boundaries [9]. Many hydraulic structure loads are produced under vertical force such as in gravity dams, water locks, and docks. The current study focuses on hydraulic structures dominated by a vertical force. The Boussinesq stress solution to a semi-infinite elastic mechanics space body under a vertical concentrated load is widely used in soil mechanics calculation [10]. This study combines 


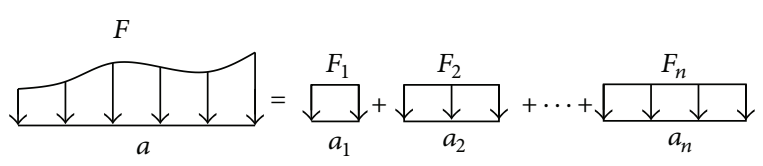

Figure 1: Decomposing the structure of a load.

L'Hospital's rule and the Boussinesq equation to derive the formula for the selection principle of foundation boundaries.

\section{Methodology}

This study investigates the problem of selecting a finite element numerical model and a foundation boundary scope for hydraulic structures under a vertical load such as gravity dams and sluices. The load of hydraulic structures on the foundation consists of nonlinear vertical distributed loads resulting from a nonlinear structure shape, nonlinear materials, and construction factors [11]. It can be decomposed into numerous distributed loads with different sizes (Figure 1). The uneven distribution of loads has different effects on foundation depth. The Boussinesq stress solution to semi-infinite elastic mechanics space body under a vertical concentrated load is widely used in soil mechanics calculation, particularly when the calculation point stress of the foundation is approximately zero. The coordinate of the point can determine the foundation boundaries of a hydraulic numerical simulation model. The formula, however, suffers from a singular point problem [12] near the ground. In this chapter, formula derivation is presented in three parts. Section 2.1 introduces the Boussinesq foundation stress formula for vertical concentrated loads. Section 2.2 describes the singular point problem near the ground and proposes solutions to this problem. Section 2.3 derives the stress formula under distributed loads with a parameter width of $a$ and determines the coordinates of a point in which stress is approximately zero to obtain the foundation boundaries for an elastic numerical simulation model of a hydraulic structure.

2.1. Introduction to Common Formulas. A hydraulic structure load on a semi-infinite elastic foundation consists of different continuous vertical distributed forces, which are decomposed into concentrated forces. Stress equations based on the half-space problem and the plane elastic mechanics problem provided by Boussinesq satisfy the objective of the aforementioned analysis problem.

Suppose that a half-space has no body force and the normal concentrated force $P$ on the plane boundary has a value of $F$. Figure 2 shows the distribution map of a load. This problem is axisymmetric, and the coordinate origin point is the center of $P$. The stress boundary condition is $\left(\sigma_{z}\right)_{z=0, y \neq 0}=$ $0,\left(\tau_{z r}\right)_{z=0, r \neq 0}=0$. The solution is described as follows:

$$
\begin{aligned}
& \sigma_{x} \\
& =\frac{3 F}{2 \pi}\left\{\frac{x^{2} z}{R^{5}}+\frac{1-2 \mu}{3}\left[\frac{1}{R(R+z)}-\frac{(2 R+z) x^{2}}{(R+z) R^{5}}-\frac{z}{R^{3}}\right]\right\},
\end{aligned}
$$

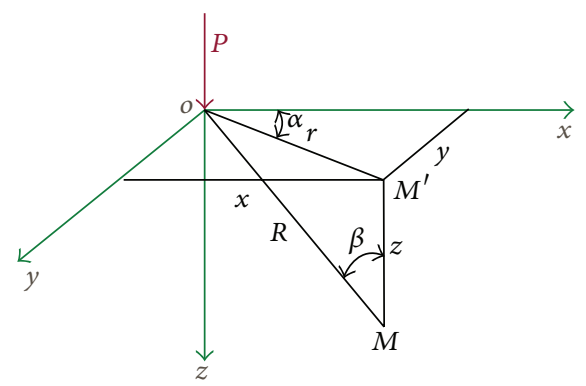

FIGURE 2: Distribution map of a load.

$$
\begin{gathered}
\sigma_{y} \\
=\frac{3 F}{2 \pi}\left\{\frac{y^{2} z}{R^{5}}+\frac{1-2 \mu}{3}\left[\frac{1}{R(R+z)}-\frac{(2 R+z) y^{2}}{(R+z) R^{5}}-\frac{z}{R^{3}}\right]\right\}, \\
\sigma_{z}=\frac{3 F}{2 \pi} \frac{z^{3}}{R^{5}} .
\end{gathered}
$$

In (1), (2), and (3), $\sigma_{x}$ is the stress in the direction of $x, \sigma_{y}$ is the stress in the direction of $y, \sigma_{z}$ is the stress in the direction of $z, \mu$ is Poisson's ratio of the foundation, and $F$ is the amount of the force.

The Boussinesq solution focuses on axisymmetric problems. The stress of hydraulic structure load on a foundation is an axisymmetric problem, wherein axes $x$ and $y$ are replaceable when studying finite element boundaries. Equations (1), (2), and (3) show that 3D stresses are proportional to the square root of length factors. The vertical stress $\left(\sigma_{z}\right)$ on the foundation is critical because it is the main factor that results in the settlement of buildings [13]. Therefore, additional research on vertical stress $\left(\sigma_{z}\right)$ to derive the foundation boundary of the finite element model is reasonable.

2.2. Singular Point of Common Formulas. Saint-Venant's principle in elastic mechanics states that if a force system acting on a small elastic surface is replaced by another equivalent static force, then the effects on the stress and strain of this region are significant, whereas the effect on distance is negligible. The accuracy of the Boussinesq formula is high when the calculation point is far from the ground. By contrast, the vertical force increases rapidly when the calculation point is near load areas, which contradicts actual situations. Thus, the point load formula is no longer applicable near the dam and requires correction. The vertical stress concentration problem near the dam area under a point load $[14,15]$ can be proven as follows:

$$
\begin{array}{r}
\left.\lim \sigma_{z}\right|_{z \rightarrow 0}=\lim _{z \rightarrow 0} \frac{3 F}{2 \pi z^{2}\left[1+(r / z)^{2}\right]^{5 / 2}} \\
=\lim _{z \rightarrow 0} \frac{3 F}{2 \pi} \frac{1 / z^{2}}{\left[1+\left(r^{2} / z^{2}\right)\right]^{5 / 2}}, \text { when } \\
u=\frac{1}{z^{2}},\left.\quad \lim \sigma_{z}\right|_{z \rightarrow 0}=\lim _{u \rightarrow \infty} \frac{u}{\left(1+r^{2} u\right)^{5 / 2}} .
\end{array}
$$


Given the randomness of $x, y$, and $z$ when they satisfy the function of

$$
\begin{aligned}
& x^{2}+y^{2}=r^{2}=\frac{1}{u^{2}}, \text { then } \\
& \left.\lim \sigma_{z}\right|_{z \rightarrow 0}=\lim _{u \rightarrow \infty} \frac{u}{\left(1+\left(1 / u^{3}\right)\right)^{5 / 2}}=\infty
\end{aligned}
$$

In the elastomeric hypothesis, the existence of stress concentration indicates the presence of a plastic expansion area. If such area exists, then the elastomeric hypothesis is contradicted. Newton solved the gravity concentration problem of two close particles by considering each particle as a sphere with a radius. To avoid stress concentration, the point load is changed to a line load with a certain length.

In Mathematical Principles and Natural Philosophy, Newton explained the law of gravitation of objects as follows. When the distance between two particles tends to be "infinitely small," the particles are regarded as a sphere with a radius [16], and consequently, the range of the integral of the force between two objects in space can be calculated. When the particles are close to each other, the Boussinesq point stress formula presents the same problem; that is, $G\left(M m / r^{2}\right) \rightarrow \infty$. Thus, particle assumption is no longer true. Based on the definition of a particle provided in Newton's law of universal gravitation, the concept of a point load is relative and can be calculated based on the particle formula when two objects are far from each other. Based on this principle, the span of a load is regarded as zero when the load is far from the dam. Newton used calculus to solve problems of increasing gravity in two particles with a small distance between them. The Boussinesq vertical stress $\sigma_{z} \rightarrow$ $\infty$ when the calculation point is near the load. Consequently, the point load near the dam should be changed to a line load with a certain length.

2.3. Deriving the New Equation. To avoid the problem of stress concentration near the ground, the aforementioned method of changing the point load to a distributed load is used to derive the correction formula for the plane and space problems. The modified formula is then used to analyze the influence of the foundation scope. When the calculation point is far from the load areas, its correction width can be ignored based on Saint-Venant's principle. By contrast, when the calculation point is close to the load areas, its correction width cannot be ignored. The following sections discuss the plane and space problems. The vertical stress formula for different depths under the effect of a point load is corrected and the function of load width parameter $a$ and depth $l$ is proposed. The relative error between the original and the corrected formula, as well as the influence of the foundation scope, is then analyzed.

2.3.1. The Plane Problem. Figure 3 shows the diagram of the plane problem. The point of the load is stipulated as the original point. Based on (3), for each point on line $z=z_{0}$, the vertical stress of point $M$ that is strictly under the original point is maximal. When the vertical stress of point $M \sigma_{z} \rightarrow$

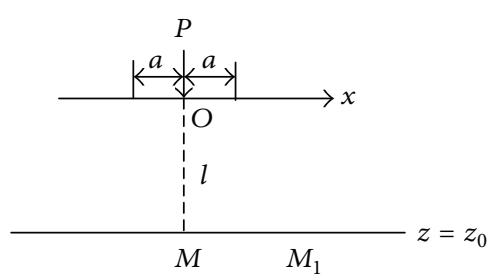

FIGURE 3: Load distribution map of the plane problem.

0 , the vertical stress of all points on line $z=z_{0}$ tends to be zero. In this research, the vertical stress $\left(\sigma_{z}\right)$ of point $M$ whose distance from the original point is $l$ is studied. The loads of hydraulic structures that are acting on the foundation are superposed by successive point loads of different sizes. The influence scope of an arbitrary point load can then be analyzed. Based on the preceding analysis, the point load is changed to a distributed load with a width of $2 a$. The following section provides the hypothetical function of width parameter $a$ and the depth of calculated point $l$.

(1) Determining the Physical Parameters. According to SaintVenant's principle, the farther the calculated point from the load areas, the less the error caused by the correction width parameter $2 a$ of the distributed load and the original point load. Meanwhile, the closer the calculated point is from the load areas, the larger the width parameter $2 a$ should be.

Assuming that the existence of critical depth $l_{0}$ satisfies $l \geq l_{0}$, parameter $a$ approximates zero and the load can be considered as a point load. By contrast, when $l<l_{0}$, the load should be considered as a distributed load. Observed through continuity, a tapering function between $l$ and $a$ exists.

Assuming that parameter $a$ is inversely proportional to $l$, then

$$
a=\frac{k}{l}
$$

The three situations of the calculated point depth $l$ are as follows.

(1) The calculation point is far from the surface with $l_{1} / a \rightarrow \infty$.

(2) The calculation point is close to the surface with $a \neq 0$, $a / l_{2} \rightarrow \infty$.

(3) The moderate area is $l_{2}<l<l_{1}$. A constant $\varepsilon>0$ exists, which ensures that $0<|l / a-N|<\varepsilon$, where $N$ is a positive integer.

(2) Correction Formula and Comparison. The following formulas correct the vertical stress formula for the three situations of the calculation point depth $l$, respectively, and compare precision before and after correction. 
(1) $l$ is extremely large; thus, $l / a \rightarrow \infty$ :

$$
\begin{gathered}
d \sigma_{z}=\frac{3 \sigma z^{3} d x}{2 \pi\left(x^{2}+z^{2}\right)^{5 / 2}} \\
\sigma_{z}=\int_{-a}^{a} d \sigma_{z}=\frac{3 \sigma l^{3}}{2 \pi} \int_{-a}^{a} \frac{d x}{\left(x^{2}+l^{2}\right)^{5 / 2}}
\end{gathered}
$$

with $x=l \tan \alpha$,

$$
\begin{gathered}
\sigma_{z}=\frac{3 \sigma}{2 \pi l} \int_{-\arctan (a / l)}^{\arctan (a / l)} \cos ^{3} \alpha d \alpha \\
=\left.\frac{3 \sigma}{\pi l}\left(\sin \alpha-\frac{1}{3} \sin ^{3} \alpha\right)\right|_{0} ^{\arctan (a / l)}, \\
\sigma_{z}=\frac{3 \sigma}{\pi l}\left(\sin \arctan \frac{a}{l}-\frac{1}{3} \sin ^{3} \arctan \frac{a}{l}\right),
\end{gathered}
$$

where $\sigma=F / 2 a$,

$$
\sigma_{z}=\frac{3 F}{2 \pi l a}\left(\sin \arctan \frac{a}{l}-\frac{1}{3} \sin ^{3} \arctan \frac{a}{l}\right) .
$$

The result of the original formula is $\sigma_{z 1}=3 F / 2 \pi l^{2}$.

The relative error is

$$
\begin{aligned}
& \left|\frac{\sigma_{z}-\sigma_{z 1}}{\sigma_{z 1}}\right| \\
& =\mid\left((3 F / 2 \pi l a)\left(\sin \arctan (a / l)-(1 / 3) \sin ^{3} \arctan (a / l)\right)\right. \\
& \left.-3 F / 2 \pi l^{2}\right) \mid \cdot\left(3 F / 2 \pi l^{2}\right)^{-1} \\
& =\lim _{a / l \rightarrow 0}\left|\frac{(3 F / 2 \pi l a)\left(a / l-a^{3} / 3 l^{3}\right)-3 F / 2 \pi l^{2}}{3 F / 2 \pi l^{2}}\right| \\
& =\lim _{a / l \rightarrow 0}\left|\frac{(3 F / 2 \pi)\left(1 / l^{2}-a^{2} / 3 l^{4}\right)-3 F / 2 \pi l^{2}}{3 F / 2 \pi l^{2}}\right| \\
& =\lim _{a / l \rightarrow 0}\left|\left(1-\frac{a^{2}}{3 l^{2}}\right)-1\right|=0 \text {. }
\end{aligned}
$$

The error is merely $a^{2} / 3 l^{2}$. Given that $l / a \rightarrow \infty$, the error is approximately zero, which illustrates that the Boussinesq solution has good applicability. Hence, the error caused by the width of the distributed load can be ignored.

(2) $l$ is very small; thus, $a \neq 0, a / l \rightarrow \infty$. The following statements prove that the stress concentration problem can be solved using (6).

The integration results of the distributed load are as follows:

$$
\begin{aligned}
& \sigma_{z}=\frac{3 F}{2 \pi l a}\left(\sin \arctan \frac{a}{l}-\frac{1}{3} \sin ^{3} \arctan \frac{a}{l}\right), \text { with } \\
& \frac{a}{l} \rightarrow \infty, \quad \lim \sigma_{z}=\frac{3 F}{2 \pi l a}\left(1-\frac{1}{3}\right)=\frac{F}{\pi k} .
\end{aligned}
$$

Thus, $\sigma_{z}$ is bounded. In fact, as long as $l \times a$ is bounded, no singular point will exist.

Apply the Taylor series at $x=0$; then

$$
\begin{gathered}
\sin (x)=x-\frac{x^{3}}{6}+o\left(x^{3}\right), \\
\arctan (x)=x-\frac{x^{3}}{3}+o\left(x^{3}\right),
\end{gathered}
$$

$$
\sigma_{z}=\frac{3 F}{2 \pi l a}\left(\frac{a}{l}-\frac{5 a^{3}}{6 l^{3}}\right)+o\left(\frac{a}{l}\right)^{3}=\frac{3 F}{2 \pi l^{2}}-\frac{5 F a^{2}}{4 \pi l^{4}} .
$$

The result of the original formula is $\sigma_{z 1}=3 F / 2 \pi l^{2}$. The relative error is

$$
\begin{aligned}
\left|\frac{\sigma_{z}-\sigma_{z 1}}{\sigma_{z 1}}\right| & =\left|\frac{3 F / 2 \pi l^{2}-3 F / 2 \pi l^{2}-5 F a^{2} / 4 \pi l^{4}}{3 F / 2 \pi l^{2}}\right| \\
& =\lim _{a / l \rightarrow \infty}\left|\frac{5 a^{2}}{6 l^{2}}\right| .
\end{aligned}
$$

Given that $a / l$ is extremely large when the calculation point is near the ground, the error caused by the width of the distributed load cannot be ignored. The stress concentration problem can be solved in this area, which demonstrates that the width of the distributed load cannot be ignored.

(3) $l$ is moderate, which corresponds to depth situation (3):

$$
\begin{aligned}
\left|\frac{\sigma_{z}-\sigma_{z 1}}{\sigma_{z 1}}\right| & \mid\left((3 F / 2 \pi l a)\left(\sin \arctan (a / l)-(1 / 3) \sin ^{3} \arctan (a / l)\right)\right. \\
& \left.-3 F / 2 \pi l^{2}\right) \mid \cdot\left(3 F / 2 \pi l^{2}\right)^{-1}, \\
& \left|\frac{\sigma_{z}-\sigma_{z 1}}{\sigma_{z 1}}\right|=\left|\frac{l}{a}\left(\operatorname{sinarctan} \frac{a}{l}-\frac{1}{3} \sin ^{3} \arctan \frac{a}{l}\right)-1\right| .
\end{aligned}
$$

Let

$$
\begin{gathered}
\frac{a}{l}=u \\
\left|\frac{\sigma_{z}-\sigma_{z 1}}{\sigma_{z 1}}\right|=\left|\left(\frac{1}{u}\right)\left(\sin \arctan u-\left(\frac{1}{3}\right) \sin ^{3} \arctan u\right)-1\right| .
\end{gathered}
$$

When searching for the intersection of $y_{1}=\sin \arctan u-$ $(1 / 3) \sin ^{3} \arctan u$ and $y_{2}=u$ using the software MATLAB, the result is

$$
u=0.106 \text {. }
$$

When $u<0.106$, the relative error and the width of the distributed load can be ignored. By contrast, when $u>$ 0.106 , the relative error cannot be ignored and the point load must be changed to a distributed load. Therefore, for a plane problem, the error caused by the width of the distributed load can be ignored only if $a / l<0.106$, and the Boussinesq point stress solution can still be used. 
2.3.2. Space Problem. In a space problem, the point load is changed to a distributed load with a radius of $a$.

(1) $a$ is the radius of the distributed load. When $l$ is extremely large, $l / a \rightarrow \infty$ :

$$
\begin{gathered}
d \sigma_{z}=\frac{3 \sigma z^{3} d x}{2 \pi\left(x^{2}+z^{2}\right)^{5 / 2}}=\frac{3 \sigma z^{3} r d r d \theta}{2 \pi\left(r^{2}+z^{2}\right)^{5 / 2}}, \\
\sigma_{z}=\int d \sigma_{z}=\frac{3 \sigma l^{3}}{2 \pi} \int_{0}^{2 \pi} d \theta \int_{0}^{a} \frac{r d r}{\left(r^{2}+z^{2}\right)^{5 / 2}} .
\end{gathered}
$$

The result of the original formula is

$$
\begin{gathered}
\sigma_{z 1}=\frac{3 F}{2 \pi l^{2}}, \\
\frac{\sigma_{z}}{\sigma_{z 1}}=-\frac{2}{3} \cdot \frac{\left(a^{2} / l^{2}+1\right)^{-3 / 2}-1}{(a / l)^{2}}, \\
\lim _{a / l \rightarrow 0} \frac{\sigma_{z}}{\sigma_{z 1}}=\lim _{a / l \rightarrow 0}-\frac{2}{3} \cdot \frac{\left(a^{2} / l^{2}+1\right)^{-3 / 2}-1}{(a / l)^{2}} \\
=\lim _{a / l \rightarrow 0}-\frac{2}{3} \cdot \frac{-(3 / 2) \cdot(a / l)^{2}}{(a / l)^{2}}=1 .
\end{gathered}
$$

The preceding equations show that when $l$ is extremely large, the error caused by the radius of the distributed load can be ignored.

(2) $l$ is extremely small, and thus $a \neq 0, a / l \rightarrow \infty$. Following the same analysis method,

$$
y=\frac{\sigma_{z}}{\sigma_{z 1}}=-\frac{2}{3} \frac{\left(a^{2} / l^{2}+1\right)^{-3 / 2}-1}{a^{2} / l^{2}} .
$$

Let

$$
\frac{a^{2}}{l^{2}}=x, \quad y=-\frac{2}{3} \frac{(x+1)^{-3 / 2}-1}{x} .
$$

Following (20), when $l$ is extremely small, the error caused by the radius of the distributed load is large.

When $a / l=0.106, y=0.986$, and the relative error is small.

\section{Applications}

The Three Gorges Dam located in Hubei Province, China, is the largest hydropower project in the world. The dam has two parts: the main building and the diversion. Three Gorges is a concrete gravity dam that is $3035 \mathrm{~m}$ long, $185 \mathrm{~m}$ high, $15 \mathrm{~m}$ wide at its crest, and $124 \mathrm{~m}$ wide at its bottom. Its installed capacity of 22.5 million $\mathrm{kW}$ ranks first in the world. In this section, the method proposed in Section 2 is first used to analyze the boundary range of the Three Gorges Dam through a numerical model. Then, the finite element numerical model of the Three Gorges Dam is established to illustrate the validity and efficiency of the proposed formulas.
3.1. Deriving Foundation Size. Hydraulic structure loads such as in gravity dams, water locks, and docks are dominated by a continuous vertical concentration. Based on the conclusions for the plane and space problems, one issue arises, that is, the calculated value of the vertical stress $\left(\sigma_{z}\right)$ at point $M$ is small when width parameter $a$ and calculation point depth value $l$ satisfy the condition of $a / l<0.106$.

The objective of the present study is to explore the boundaries of the finite element model selection problem for vertical concentration loads, which is equivalent to finding the coordinates of the points when the base stress is small. In the following section, the base boundary depth of the finite element model is analyzed using the preceding conclusions.

Because of the limit of influenced scope based on the hydraulic structure loads, so the foundation boundary belongs to the result of (11). Equation (11) shows the stress results of the definite integral as follows:

$$
\sigma_{z}=\frac{3 F}{2 \pi k}\left(\sin \arctan \frac{a}{l}-\frac{1}{3} \sin ^{3} \arctan \frac{a}{l}\right) .
$$

For this formula, various hydraulic structures have different values of $3 F / 2 \pi k$ because of structure size and material properties. When depth $l$ of the calculation point satisfies the condition

$$
a / l=0.106
$$

$\sigma_{z}$ tends to be equal to zero. Parameter $a$ is restricted by the size of the structures. Assume that width $L$ and height $H$ of hydraulic structures satisfy the equation $L=m H$. Then, the deeper $l$ is, the smaller parameter $a$ is, which results in a smaller calculation error of the Boussinesq equation. The maximum value of load length $2 a$ is the width $L$ of the structure, which corresponds to the maximum depth as follows:

$$
l_{\max }=\frac{a_{\max }}{0.106}=\frac{L}{2 * 0.106}=4.717 L=4.717 m H .
$$

When depth $l$ of the calculation point satisfies (23), vertical stress $\left(\sigma_{z}\right)$ tends to be zero, and $l$ is equivalent to the depth boundary of the finite element calculation model. Various hydraulic structures have different values for parameter $m$. The value of $m$ should be calculated for each project, and then the base depth boundary of the finite element numerical simulation model can be calculated.

The preceding conclusions can be applied to a specific project. The Three Gorges Water Conservancy Project is the largest gravity dam project in China. In this study, the depth boundary of the finite element numerical simulation model of the aforementioned project is analyzed. The Three Gorges Dam Project is $185 \mathrm{~m}$ high and $124 \mathrm{~m}$ wide. The parameter $m$ of Three Gorges is

$$
m=\frac{L}{H}=\frac{124}{185}=0.670 .
$$

Its depth boundary is $l_{\max }=4.717 \mathrm{mH}=3.16 \mathrm{H}$. 


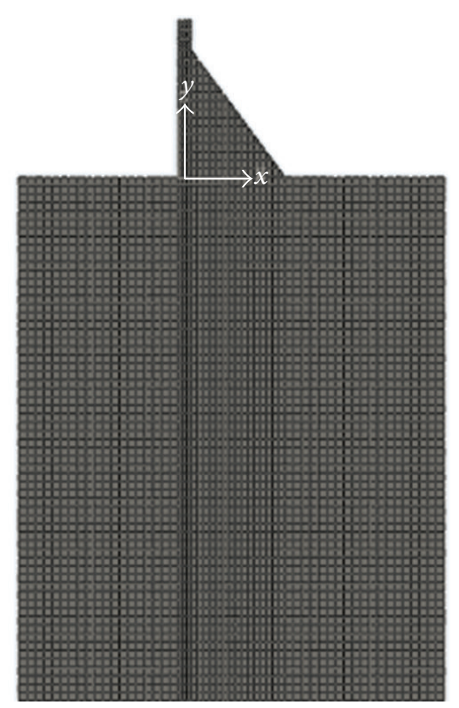

FIGURE 4: Finite element model.

TABLE 1: FEM material parameters.

\begin{tabular}{lccc}
\hline Area & $\begin{array}{c}\text { Parameter } \\
\text { modormation } \\
\text { modulus }(\mathrm{GPa})\end{array}$ & $\begin{array}{c}\text { Poisson's } \\
\text { ratio }(\mu)\end{array}$ & $\begin{array}{c}\text { Density } \\
\left(\mathrm{kg} / \mathrm{m}^{3}\right)\end{array}$ \\
\hline Dam concrete & 21 & 0.167 & 2400 \\
Foundation & 26 & 0.2 & 2450 \\
\hline
\end{tabular}

TABLE 2: FEM dimensions of the dam body.

\begin{tabular}{lcccc}
\hline Area & $\begin{array}{c}\text { Width of } \\
\text { dam crest }\end{array}$ & $\begin{array}{c}\text { Width of } \\
\text { dam bottom }\end{array}$ & $\begin{array}{c}\text { Height } \\
\text { of dam }\end{array}$ & $\begin{array}{c}\text { Slope of } \\
\text { downstream } \\
\text { surface }\end{array}$ \\
\hline Dam body & $15 \mathrm{~m}$ & $124 \mathrm{~m}$ & $185 \mathrm{~m}$ & 0.75 \\
\hline
\end{tabular}

TABLE 3: FEM dimensions of the foundation.

\begin{tabular}{lccc}
\hline Area & $\begin{array}{c}\text { Width of } \\
\text { upstream }\end{array}$ & $\begin{array}{c}\text { Dimensions } \\
\text { Width of } \\
\text { upstream }\end{array}$ & $\begin{array}{c}\text { Depth of } \\
\text { foundation }\end{array}$ \\
\hline Foundation & $185 \mathrm{~m}$ & $185 \mathrm{~m}$ & $740 \mathrm{~m}$ \\
\hline
\end{tabular}

3.2. Finite Element Numerical Simulation. The establishment of the finite element model of the Three Gorges Dam illustrates the effectiveness and accuracy of the model analysis used in this study. Figure 4 shows the finite element model of the Three Gorges Dam with the following specifications: the $x$-axis along the downstream direction, the $y$-axis along the dam axis, and the $z$-axis along the vertical direction. The finite element model is divided into 5393 units and 11102 nodes. The FEM material parameters are presented in Table 1 . The actual dimensions of the finite element model of the Three Gorges Dam are provided in Tables 2 and 3.

Based on the aforementioned calculation principle and parameter, the specific calculation results and analyses are as follows.

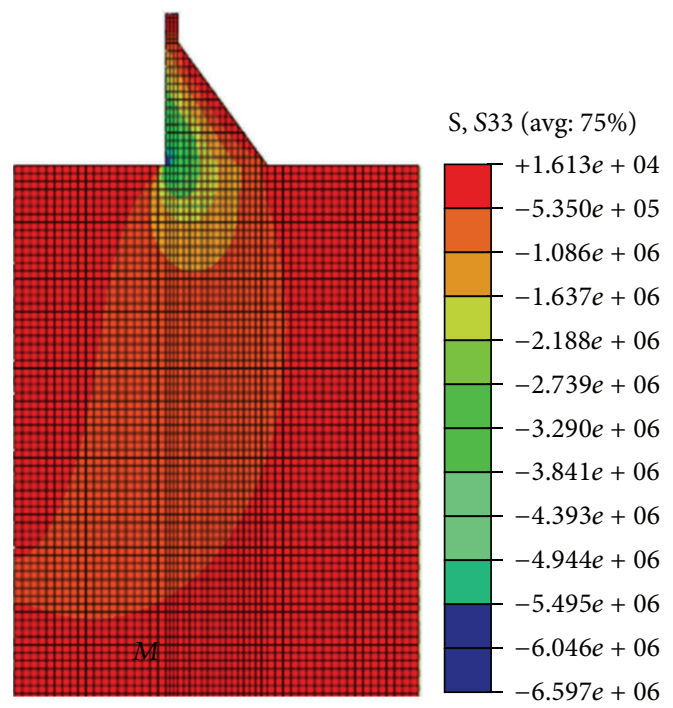

FIGURE 5: FEM vertical stress $\sigma_{z}$.

Given that the foundation and dam body have the same size and material along the $y$-axis direction, the stress results of the plane and space problems along the $y$-axis section are also the same. The following finite element model is focused on plane problems. Figure 5 illustrates the vertical stress $\left(\sigma_{z}\right)$ calculation results of the finite element model of the Three Gorges Dam. All size parameters of the finite element method are chosen for the international system of units: meters. The legend of Figure 5 is the size range of $\sigma_{z}$ (in Pascal). This figure can indicate that the foundation vertical stress $\left(\sigma_{z}\right)$ is approximately zero when depth is deeper than the depth of point $M$. The depth of point $M$ is the furthest depth of the load effect. Figure 5 shows the depth of point $M$; that is, $Z_{m}=556 \mathrm{~m}$. Dam height is $185 \mathrm{~m}$; that is, $Z_{M}=556 / 185 \mathrm{H}=$ $3.01 \mathrm{H}$.

The boundary range acquisition method is based on the Boussinesq formula. This formula is suitable for a semiinfinite body problem under a concentrated load stress. The finite element model of the Three Gorges Dam selected $1 H$ to be the foundation range upstream and downstream and $4 H$ to be the foundation depth range. According to the finite element calculation results, the foundation influence scope is limited on the loads of hydraulic structures and expanding the scope of the foundation boundary has minimal effect on calculation precision. The Boussinesq formula accurately derives the elastic mechanics formula based on a differential equation, but FEM is the solution to a partial differential equation. Moreover, numerical solution techniques are approximated through the variation method, and the error function is set to minimum values to generate a stable solution. FEM is an approximate method for solving the Boussinesq half-space problem. The two methods, which have different principles, produce slightly different results; however, they exhibit the same trend. The depth boundary defined in Section 3.1 is $Z=3.16 \mathrm{H}$. Its relative error is less than 5\%; therefore, the model exhibits high precision. Furthermore, the model proves that (23) can be used as 
a guideline for depth boundary in the numerical simulation of gravity dams.

\section{Conclusions}

The following conclusions can be drawn from this study.

(1) Drawing lessons from the small distance correction method of Newton's law of gravity, the point load is changed to a line load to solve the singular point problem near the face area. Through correct results, (23) can function as a guideline for depth boundary in establishing finite element numerical simulation models of hydraulic structures.

(2) The formula of parameter $a$ and $l$ should be researched thoroughly. In this study, dam bottom width $L$ is set to a maximum value of $2 a$. By using this method, maximum depth boundary $l_{\max }$ is achieved. If a certain magnitude for vertical stress $\left(\sigma_{z}\right)$ is set, then depth boundary can be reduced.

(3) This study only investigated the boundary of direction $z$. The boundaries of directions $x$ and $y$ should be studied further.

\section{Conflict of Interests}

The authors declare that there is no conflict of interests regarding the publication of this paper.

\section{Acknowledgments}

This work was supported by the National Natural Science Foundation of China (Grant nos. 41323001, 51139001, 51379068, 51179066, 51279052, and 51209077), the Jiangsu Natural Science Foundation (Grant nos. BK20140039 and BK2012036), and the Research Fund for the Doctoral Program of Higher Education of China (Grant nos. 20120094110005, 20120094130003, and 20130094110010).

\section{References}

[1] L. Eisenlohr, L. Király, M. Bouzelboudjen, and Y. Rossier, "Numerical simulation as a tool for checking the interpretation of karst spring hydrographs," Journal of Hydrology, vol. 193, no. 1-4, pp. 306-315, 1997.

[2] N. Moës, J. Dolbow, and T. Belytschko, "A finite element method for crack growth without remeshing," International Journal for Numerical Methods in Engineering, vol. 46, no. 1, pp. 131-150, 1999.

[3] D. V. Griffiths and P. A. Lane, "Slope stability analysis by finite elements," Geotechnique, vol. 49, no. 3, pp. 387-403, 1999.

[4] L. Simoni and B. A. Schrefler, "An accelerated algorithm for parameter identification in a hierarchical plasticity model accounting for material constraints," International Journal for Numerical and Analytical Methods in Geomechanics, vol. 25, no. 3, pp. 263-272, 2001.

[5] G. Chongshi, "Precision analysis on simulational finite element model of dam and batholith," Hydroelectric Energy, vol. 20, no. 2, pp. 13-16, 2002.
[6] D.-Y. Shi, S.-P. Mao, and S.-C. Chen, "On the anisotropic accuracy analysis of ACM's nonconforming finite element," Journal of Computational Mathematics, vol. 23, no. 6, pp. 635-646, 2005.

[7] T. H. Vu and A. J. Deeks, "Using fundamental solutions in the scaled boundary finite element method to solve problems with concentrated loads," Computational Mechanics, vol. 53, no. 4, pp. 641-657, 2014.

[8] S. Chirită and R. Quintanilla, "On Saint-Venant's principle in linear elastodynamics," Journal of Elasticity, vol. 42, no. 3, pp. 201-215, 1996.

[9] L.-C. Huang, Z.-S. Xu, and L.-C. Wang, "Constitutive equations and finite element implementation of strain localization in sand deformation," Journal of Central South University of Technology (English Edition), vol. 16, no. 3, pp. 482-487, 2009.

[10] Q. Feng, “Traveling wave solutions for the variant Boussinseq equation and the $(2+1)$-dimensional Nizhnik-Novikov-Veselov (NNV) system by $\left(\mathrm{G}^{\prime} / \mathrm{G}\right)$-expansion method," WSEAS Transactions on Mathematics, vol. 9, no. 1-3, pp. 191-200, 2010.

[11] S. R. Lan and J. S. Yang, "Nonlinear finite element analysis of arch dam .1. Constitutive relationship," Advances in Engineering Software, vol. 28, no. 7, pp. 403-408, 1997.

[12] Z. Y. Yan and H. Q. Zhang, "New explicit and exact travelling wave solutions for a system of variant Boussinesq equations in mathematical physics," Physics Letters A, vol. 252, no. 6, pp. 291296, 1999.

[13] O. Enkhtur, T. D. Nguyen, J. M. Kim, and S. R. Kim, "Evaluation of the settlement influence factors of shallow foundation by numerical analyses," KSCE Journal of Civil Engineering, vol. 17, no. 1, pp. 85-95, 2013.

[14] H. G. Georgiadis and D. S. Anagnostou, "Problems of the flamant-boussinesq and kelvin type in dipolar gradient elasticity," Journal of Elasticity, vol. 90, no. 1, pp. 71-98, 2008.

[15] A. M. Malek, H. Saadatmanesh, and M. R. Ehsani, "Prediction of failure load of R/C beams strengthened with FRP plate due to stress concentration at the plate end," ACI Structural Journal, vol. 95, no. 2, pp. 142-152, 1998.

[16] P. Fara, "Philosophiae naturalis principia mathematica (Mathematical Principles of Natural Philosophy)," Nature, vol. 499, no. 7457, p. 153, 2013. 


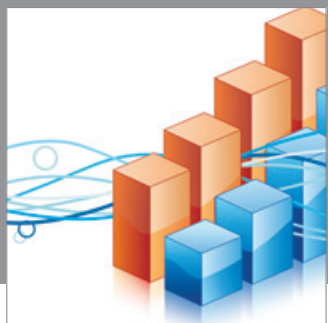

Advances in

Operations Research

mansans

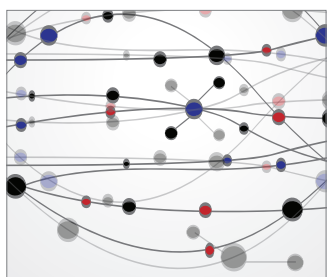

The Scientific World Journal
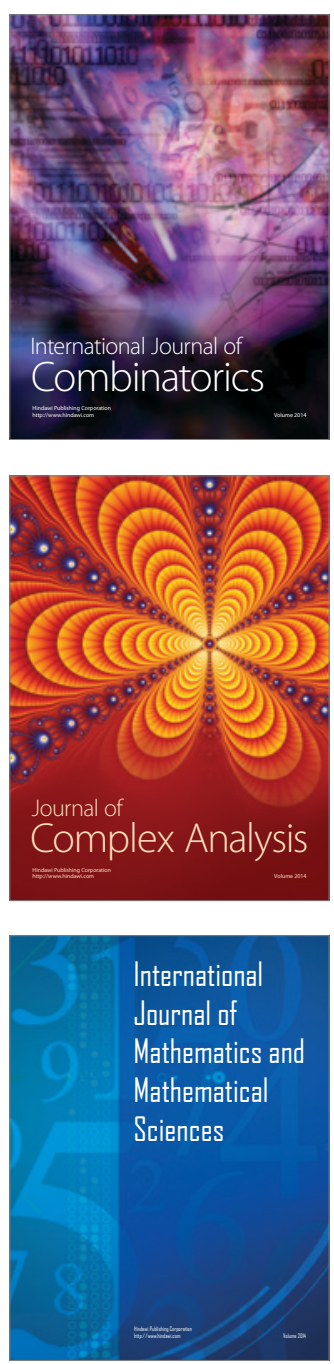
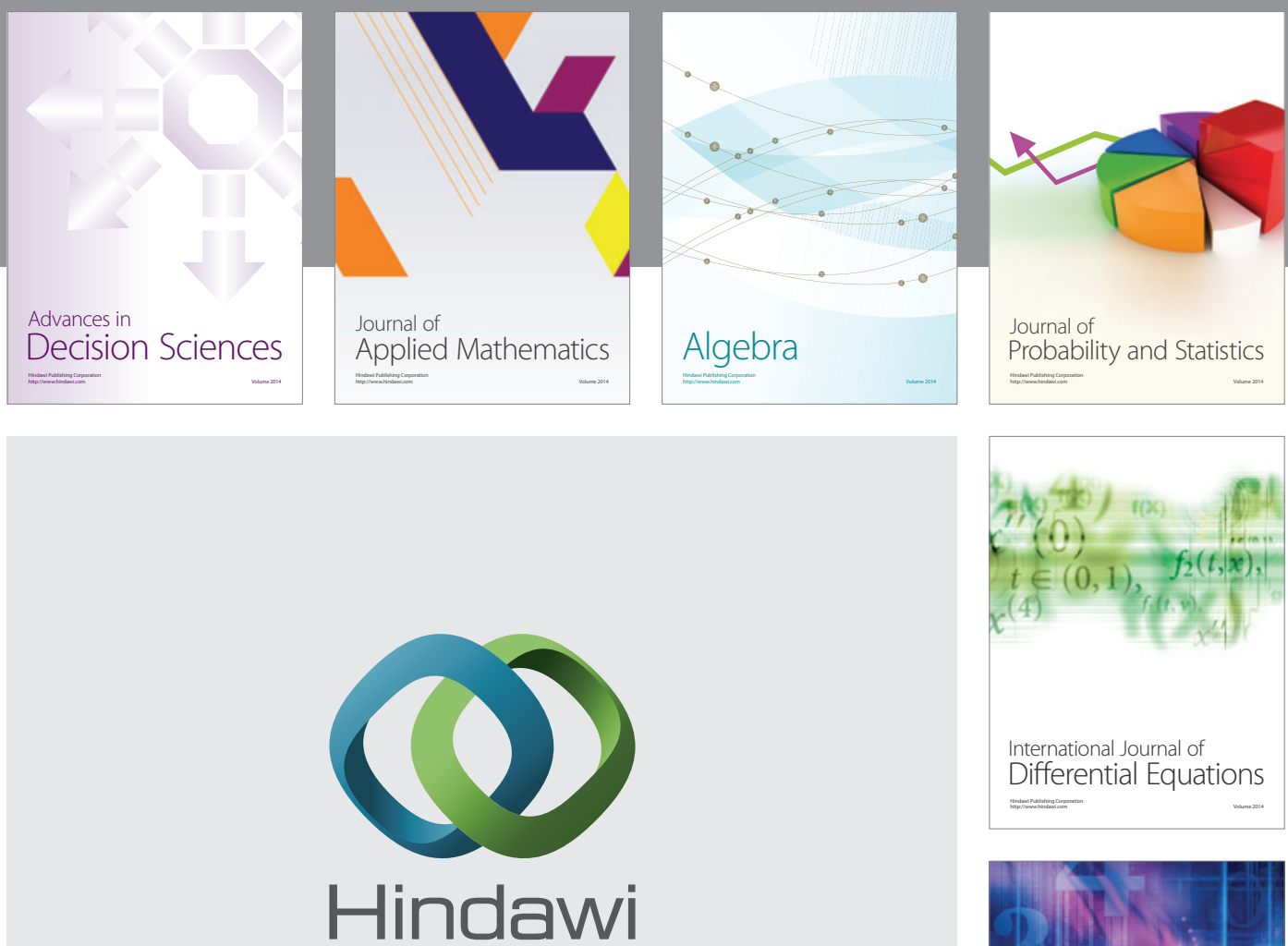

Submit your manuscripts at http://www.hindawi.com
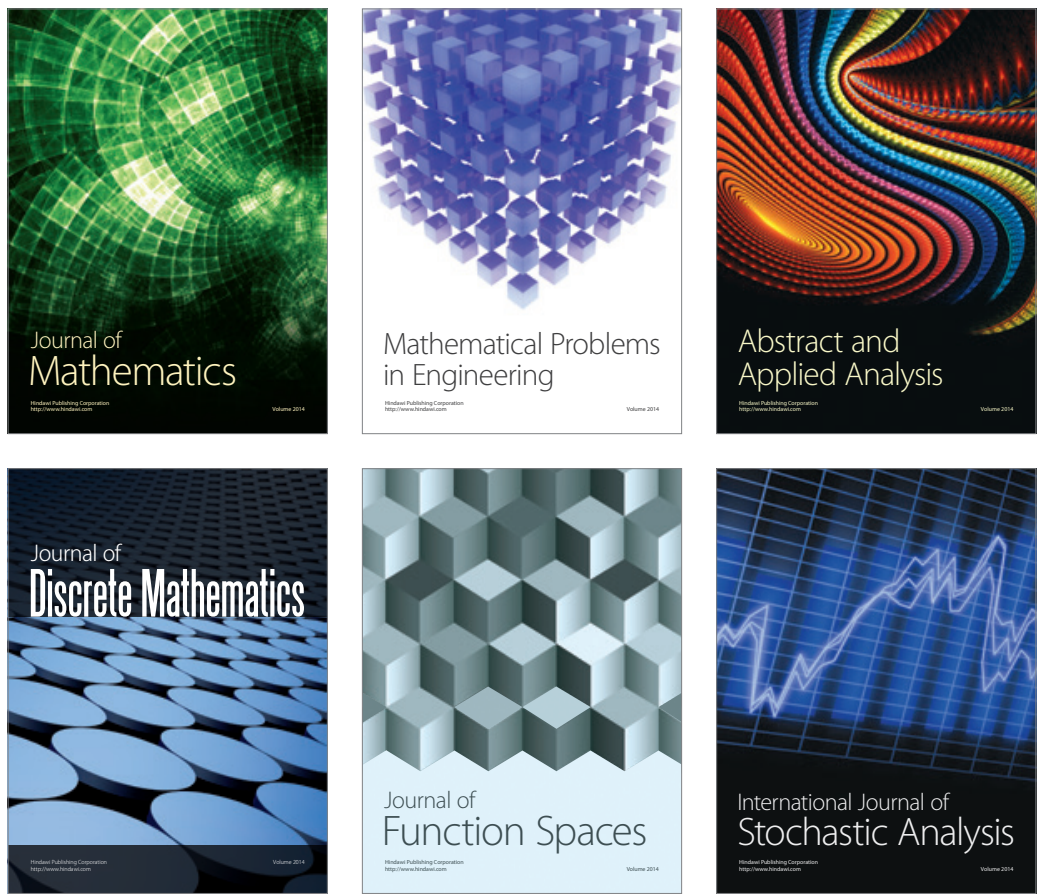

Journal of

Function Spaces

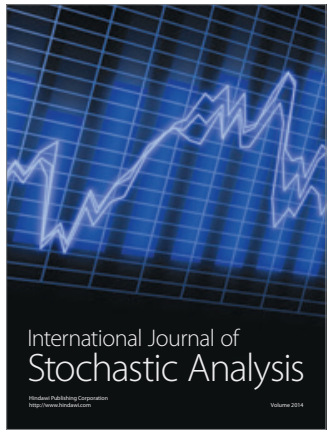

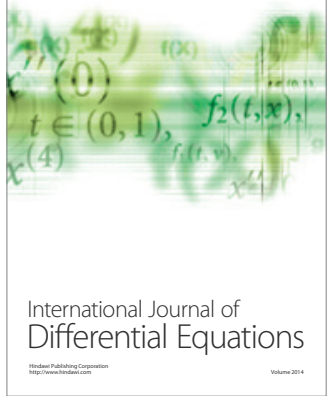
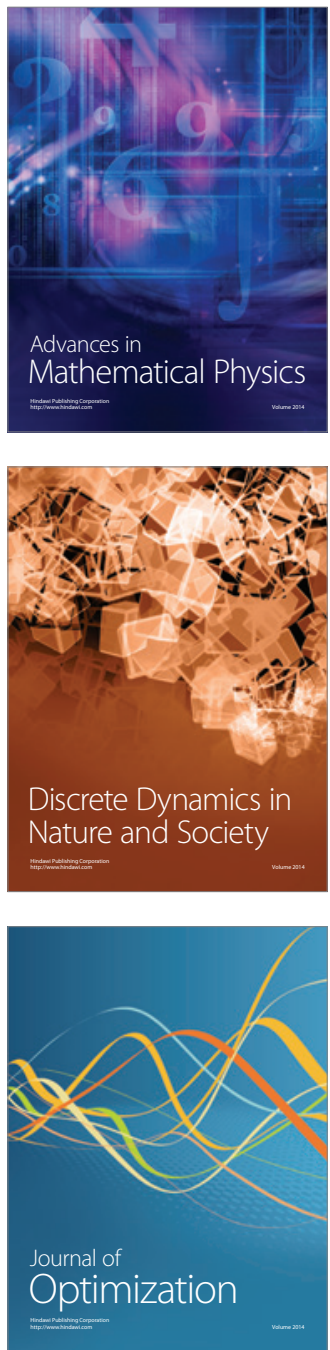